\title{
Improved reporting of overuse injuries and health problems in sport: an update of the Oslo Sport Trauma Research Center questionnaires
}

\author{
Benjamin Clarsen (D) , Roald Bahr, ${ }^{1}$ Grethe Myklebust, ' Stig Haugsboe Andersson, ${ }^{1}$ \\ Sean lain Docking (D), ${ }^{2}$ Michael Drew, ${ }^{3}$ Caroline F Finch (D) , \\ Lauren Victoria Fortington (10) , Joar Harøy (10) , ${ }^{4}$ Karim M Khan (D) , ${ }^{5}$ Bill Moreau, ${ }^{6,7}$ \\ Isabel S Moore (D) , ${ }^{8}$ Merete Møller, ${ }^{9}$ Dustin Nabhan (D) 1,10 \\ Rasmus Oestergaard Nielsen (D) , ${ }^{11}$ Kati Pasanen (D) , ${ }^{12,13}$ Martin Schwellnus, ${ }^{14}$ \\ Torbjørn Soligard (D) , ${ }^{15}$ Evert Verhagen (D) ${ }^{16}$
}

For numbered affiliations see end of article.

\section{Correspondence to} Dr Benjamin Clarsen, Department of Sports Medicine, Norwegian School of Sport Sciences, Oslo Sports Trauma Research Center, Oslo 0806, Norway; ben.clarsen@nih.no

Accepted 28 January 2020 Published Online First 14 February 2020
Check for updates

(C) Author(s) (or their employer(s)) 2020. No commercial re-use. See rights and permissions. Published by BMJ.

To cite: Clarsen $B$

Bahr R, Myklebust G,

et al. Br J Sports Med

2020:54:390-396.

\section{ABSTRACT}

In 2013, the Oslo Sports Trauma Research Center Overuse Injury Questionnaire (OSTRC-0) was developed to record the magnitude, symptoms and consequences of overuse injuries in sport. Shortly afterwards, a modified version of the OSTRC-O was developed to capture all types of injuries and illnesses-The Oslo Sports Trauma Research Center Questionnaire on Health Problems (OSTRC-H). Since then, users from a range of research and clinical environments have identified areas in which these questionnaires may be improved. Therefore, the structure and content of the questionnaires was reviewed by an international panel consisting of the original developers, other user groups and experts in sports epidemiology and applied statistical methodology. Following a review panel meeting in October 2017, several changes were made to the questionnaires, including minor wording alterations, changes to the content of one question and the addition of questionnaire logic. In this paper, we present the updated versions of the questionnaires (OSTRC-02 and OSTRC- $-\mathrm{H} 2$ ), assess the likely impact of the updates on future data collection and discuss practical issues related to application of the questionnaires. We believe this update will improve respondent adherence and improve the quality of collected data.

\section{INTRODUCTION}

The Oslo Sports Trauma Research Center (OSTRC) Overuse Injury Questionnaire was developed to address challenges which arise when using traditional sports injury surveillance methods to document the epidemiology of overuse injuries. ${ }^{1}$ Traditionally, most injury surveillance studies used time loss from sport as the primary criterion for defining the occurrence of injury and the duration of time lost as a surrogate measure of injury severity. ${ }^{2}$ This approach underestimates the full impact of overuse injuries because athletes with an overuse injury can often continue to train and compete despite persistent injury-associated symptoms and limitations. ${ }^{3}$ The OSTRC Overuse Injury Questionnaire contains four domains which seek to evaluate the consequences of overuse injuries on athletes: (1) sports participation, (2) training volume, (3) sports performance and (4) pain (table 1, first column). By administering the questionnaire at regular intervals (eg, weekly), clinicians and researchers are able to monitor how the consequences of overuse injury change over time.

The OSTRC Overuse Injury Questionnaire was initially developed to collect information on overuse injuries in specific, predefined, anatomical areas. However, it quickly became apparent that the approach was not only suited to recording overuse injuries-athletes may also continue to participate after sustaining acute injuries or while suffering illness. ${ }^{4}$ Therefore, a modified version of the overuse injury questionnaire was developed that allowed athletes to record all types of health problems-the OSTRC Questionnaire on Health Problems. ${ }^{5}$ In this version, the four key questions referred to all types of health problems (table 2, first column), and if an athlete reported a problem, he/she then had to provide additional information such as the type of problem and its location or main symptoms.

The OSTRC overuse injury and health problems questionnaires have been widely cited and adopted in sports injury research since their initial publications in 2013 and 2014. A citation search performed in March 2019 using Thompson-Reuters Web of Science database identified 254 citations of the questionnaires, including 59 and 14 studies that used the overuse injury and health problem questionnaires, respectively, to collect data.

In addition to its research applications, the OSTRC health problems questionnaire has also gained popularity as a clinical monitoring tool to evaluate health trends and ensure timely care to athletes in a range of elite sports organisations. Users include, among others, the Norwegian, US, Australian and Dutch Olympic programmes and the Norwegian, US, Dutch and German Paralympic programmes.

Due to this successful uptake, user groups from a range of research and clinical environments gained experience using the OSTRC questionnaires and identified several issues requiring consideration. To address these issues, which ranged from wording clarification to data analysis principles, the original 
Table 1 Original and updated versions of the OSTRC-0 questionnaire, with changes highlighted in red

\begin{tabular}{|c|c|}
\hline \multicolumn{2}{|l|}{ The OSTRC-0 } \\
\hline Original version (OSTRC-0) & Updated version (OSTRC-02) \\
\hline $\begin{array}{l}\text { Please answer all questions regardless of whether or not you have problems with your } \\
\text { knees. Select the alternative that is most appropriate for you, and in the case that you } \\
\text { are unsure, try to give an answer as best you can anyway. }\end{array}$ & $\begin{array}{l}\text { Please answer all questions regardless of whether or not you have problems in } \\
\text { your(insert anatomical location here, eg, knees). Select the alternative that is most } \\
\text { appropriate for you, and in the case that you are unsure, try to answer as best you can } \\
\text { anyway. }\end{array}$ \\
\hline $\begin{array}{l}\text { The term 'knee problems' refers to pain, ache, stiffness, swelling, instability/giving way, } \\
\text { locking or other complaints related to one or both knees. }\end{array}$ & $\begin{array}{l}\text { The term '(location) problems' refers to (insert common symptoms or injury } \\
\text { consequences here, eg, pain, ache, stiffness, clicking/catching, swelling, instability/giving } \\
\text { way, locking)or other complaints related to your (location). }\end{array}$ \\
\hline $\begin{array}{l}\text { Have you had any difficulties participating in normal training and competition due to } \\
\text { knee problems during the past week? }\end{array}$ & $\begin{array}{l}\text { Have you had any difficulties participating in training and competition due to (location) } \\
\text { problems during the past } 7 \text { days? }\end{array}$ \\
\hline a. Full participation without knee problems & a. Full participation without (location) problems \\
\hline b. Full participation, but with knee problems & b. Full participation, but with (location) problems \\
\hline c. Reduced participation due to knee problems & c. Reduced participation due to (location) problems \\
\hline a. No reduction & a. No modification \\
\hline b. To a minor extent & b. To a minor extent \\
\hline c. To a moderate extent & c. To a moderate extent \\
\hline d. To a major extent & d. To a major extent \\
\hline \multicolumn{2}{|l|}{ e. Cannot participate at all } \\
\hline Question 3 & Question 3-Performance \\
\hline To what extent have knee problems affected your performance during the past week? & $\begin{array}{l}\text { To what extent have (location) problems affected your performance during the past } 7 \\
\text { days? }\end{array}$ \\
\hline a. No effect & a. No effect \\
\hline b. To a minor extent & b. To a minor extent \\
\hline c. To a moderate extent & c. To a moderate extent \\
\hline c. Moderate pain & c. Moderate pain \\
\hline d. Severe pain & d. Severe pain \\
\hline
\end{tabular}

OSTRC-0, Oslo Sports Trauma Research Center Overuse Injury Questionnaire.

developers of the questionnaire initiated a review process in August 2017 that included consulting with an international panel of researchers and clinicians who represented key user groups. The process included a review panel meeting in Oslo on 3 and 4 October 2017.

In this paper, we summarise the topics discussed during the meeting and introduce several changes to the wording, structure and logic of the original questionnaires. We also analyse new and previously collected data to illustrate the impact of the changes.

We refer to the OSTRC Overuse Injury Questionnaire and OSTRC Questionnaire on Health Problems collectively as the OSTRC questionnaires except where it is necessary to specify one in particular. In those cases, we use the abbreviation OSTRC-O and OSTRC-H. ${ }^{6}$ We use the suffix ' 2 ' to specify the updated versions of the questionnaires (ie, OSTRC-O2 and OSTRC-H2).

\section{QUESTIONNAIRE REVIEW PROCEDURE}

Review panel members were identified by the primary author $(\mathrm{BC})$ based on their experience using the OSTRC questionnaires for research or clinical purposes or their expertise in epidemiological and applied statistics methodology in the sports medicine context. Prior to the meeting, the primary author had informal discussions with all panel members to establish the meeting agenda. Panel members who were unable to attend the meeting $(\mathrm{n}=5)$ provided a written summary of their positions on each agenda item. Following the meeting, a detailed summary was distributed to the entire panel, including the proposed changes to the questionnaire wording, logic and response categories. The panel agreed unanimously on all changes and contributed as authors of this manuscript.

\section{CHANGES TO THE OSTRC QUESTIONNAIRES}

We considered each of the four key OSTRC questions in detail and discussed the need for changes to the wording, logic and response categories. Proposed changes were categorised as minor or more substantial. A more substantial change was defined as one where the panel agreed that such a change required validation.

\section{Minor (inconsequential) wording changes}

The panel noted several areas of ambiguity and inconsistency between questions in the original questionnaires and agreed to 
Table 2 Original and updated versions of the OSTRC-H questionnaire, with changes highlighted in red

The OSTRC-H
Original version (OSTRC-H)
Please answer all questions regardless of whether or not you have experienced health
problems in the past week. Select the alternative that is most appropriate for you, and in
the case that you are unsure, try to give an answer as best you can anyway.
If you have several illness or injury problems, please refer to the one that has been your
worst problem this week. You will have a chance to register other problems at the end of
the questionnaire.
the questionnaire.

\section{Question 1}

Have you had any difficulties participating in normal training and competition due to injury, illness or other health problems during the past week?

a. Full participation without health problems

b. Full participation, but with injury/illness

c. Reduced participation due to injury/illness

d. Cannot participate due to injury/illness

\section{Question 2}

To what extent have you reduced you training volume due to injury, illness or other health problems during the past week?

a. No reduction

b. To a minor extent

c. To a moderate extent

d. To a major extent

e. Cannot participate at all

\section{Question 3}

To what extent has injury, illness or other health problems affected your performance during the past week?

a. No effect

b. To a minor extent

c. To a moderate extent

d. To a major extent

e. Cannot participate at all

Question 4

To what extent have you experienced symptoms/health complaints during the past week?

a. No symptoms/health complaints

b. To a mild extent

c. To a moderate extent

d. To a severe extent
Updated version (OSTRC-H2)

Please answer all questions regardless of whether or not you have experienced health problems in the past 7 days. Select the alternative that is most appropriate for you, and in the case that you are unsure, try to answer as best you can anyway.

A health problem is any condition that you consider to be a reduction in your normal state of full health, irrespective of its consequences on your sports participation or performance, or whether you have sought medical attention. This may include, but is not limited to, injury, illness, pain or mental health conditions.

If you have several health problems, please begin by recording your worst problem in the past 7 days. You will have a chance to register other problems at the end of the questionnaire.

Question 1-Participation

Have you had any difficulties participating in training and competition due to injury, illness or other health problems during the past 7 days?

a. Full participation without health problems

b. Full participation, but with a health problem

c. Reduced participation due to a health problem

d. Could not participate due to a health problem

Question 2-Modified training/competition

To what extent have you modified your training or competition due to injury, illness or other health problems during the past 7 days?

a. No modification

b. To a minor extent

c. To a moderate extent

d. To a major extent

Question 3-Performance

To what extent has injury, illness or other health problems affected your performance during the past 7 days?

a. No effect

b. To a minor extent

c. To a moderate extent

d. To a major extent

Question 4-Symptoms

To what extent have you experienced symptoms/health complaints during the past 7 days?

a. No symptoms/health complaints

b. To a mild extent

c. To a moderate extent

d. To a severe extent

OSTRC-H, Oslo Sports Trauma Research Center Questionnaire on Health Problems.

make minor changes to the questionnaire instructions and to the wording of all four questions. These changes included replacing 'the past week' with 'the past 7 days', replacing 'cannot' with 'could not' and adding titles to differentiate the questions (tables 1 and 2, second columns).

In the OSTRC-H2, we replaced 'injury/illness' with 'health problem' in the instructions, questions and response categories. In the OSTRC-H2 instructions, we included the following definition of health problem: 'A health problem is any condition that you consider to be a reduction in your normal state of full health, irrespective of its consequences on your sports participation or performance, or whether you have sought medical attention. This may include, but is not limited to, injury, illness, pain or mental health conditions.'

This definition is consistent with the International Olympic Committee consensus statement on methods for recording and reporting epidemiological data on injury and illness in sports. ${ }^{7}$
The original OSTRC-O was published using only the knee, shoulder and low back as example areas. However, some readers appear to have misinterpreted this as meaning that the OSTRC-O is a specific knee, shoulder and low back questionnaire. Therefore, we made minor changes to the OSTRC-O template to clarify that the questionnaire is applicable to any anatomical region (table 1 , second column).

\section{More substantial changes}

We recognised that reducing training volume is only one way in which an athlete can modify their normal sports participation in response to a health problem. The original question may have missed other common modifications such as reduced intensity, changes in the type of training (eg, cycling instead of running) or changing roles in a team (tactical/positional). To address this, we changed the wording of question 2 ; instead of asking about 
Table 3 Comparison between the responses to the old and new wording of question 2

\begin{tabular}{lllllll}
\hline & \multicolumn{5}{l}{$\begin{array}{l}\text { New wording } \\
\text { Training or competition modification }\end{array}$} \\
\cline { 3 - 7 } & & 2a & 2b & 2c & 2d & 2e \\
\hline Old & $2 \mathrm{a}$ & 52 & 15 & 2 & 0 & 0 \\
wording & $2 \mathrm{~b}$ & 11 & 14 & 1 & 0 & 0 \\
Training & $2 \mathrm{c}$ & 0 & 1 & 2 & 0 & 0 \\
reduction & $2 \mathrm{~d}$ & 0 & 1 & 1 & 9 & 0 \\
& $2 \mathrm{e}$ & 0 & 0 & 0 & 1 & 8 \\
\hline
\end{tabular}

Data were collected from 90 athletes over 13 weeks; a total of 596 questionnaire responses were collected, of which 118 included a health problem.

Column and row headings $2 \mathrm{a}-\mathrm{e}$ represent response categories to question 2 as shown in (tables 1 and 2).

In this analysis, we did not apply gatekeeper logic and thus included a fifth response category (could not participate at all) to the new question.

the extent to which an athlete has reduced their training volume, the revised question asks about the extent to which athletes have modified their training or competition. To align with this change, the word 'normal' was removed from question 1.

Athletes may answer differently when asked about modified training or competition, compared with reduced training volume. To assess the consequences of the change in wording, for a period of 13 weeks, we included both questions simultaneously in the ongoing registry of three Dutch National Olympic programmes (ie, water polo, equestrian sports and baseball); these programmes, included 90 athletes familiar with the OSTRC-H. To assess the level of agreement between responses to both questions, we calculated Cohen's kappa using equal weights (table 3 ). The kappa coefficient was 0.55 which suggests substantial differences. However, the main inconsistencies between versions 1 and 2 occurred for the least severe health problems (ie, those with little or no consequences on training). These differences were consistent with our reasoning to modify the questioning.

\section{Changes to questionnaire logic and answer categories}

As questions 2-4 are only relevant for athletes who have a health problem and continue to participate in training and competition, we propose a new 'gatekeeper' logic that can be applied to question 1. Using this logic:

- If an athlete selects the first answer option 'full participation without health problems', all further questions are redundant. In this case, a total severity score of 0 is assigned and the questionnaire is complete.

- If an athlete selects the fourth answer option 'could not participate due to a health problem', questions 2-4 are redundant. In this case, a total severity score of 100 is assigned. The athlete continues directly to additional questions researchers may apply to the questionnaire classify the reported health problem.

This logic will reduce unnecessary responder burden by ensuring athletes only receive questions relevant to their current health state. However, it is important to note that in the past, when respondents were expected to complete all four key questions, their responses were not always consistent. For example, an athlete may have reported 'reduced participation due to a health problem' in question 1 , then 'cannot participate at all' in question 2 or 3 . Consequently, for consistency and clarity, we removed the response category 'cannot participate at all' from questions 2 and 3 . We recommend that for these questions, the
Table 4 Comparison of the number of injuries and illnesses identified when gatekeeper logic is and is not applied to question 1

\begin{tabular}{llll}
\hline & No logic & Gatekeeper logic & $\begin{array}{l}\text { Difference } \\
(\%)\end{array}$ \\
\hline All problems & & & \\
$\quad$ Injuries & 3460 & 3045 & 12.0 \\
$\quad$ Illnesses & 1857 & 1574 & 15.2 \\
$\begin{array}{l}\text { Substantial problems } \\
\quad \text { Injuries }\end{array}$ & 1245 & 1236 & \\
$\quad$ Illnesses & 1049 & 1024 & 0.7 \\
Time loss problems* & & & 2.4 \\
$\quad$ Injuries & 1295 & 1244 & 3.9 \\
$\quad$ Illnesses & 1251 & 1171 & 6.4 \\
\hline
\end{tabular}

Data are based on a convenience sample of 13888 OSTRC-H responses from elite Norwegian athletes.

*Identified using an additional question that asked respondents how many days they were unable to train or compete due to that health problem in the past 7 days.

values to calculate the severity score are aligned with question 1 and 4 (ie, $A=0, B=8, C=17, D=25$ ) (readers are directed to Ref. ${ }^{1}$ for a full explanation of the OSTRC severity score).

\section{Consequences of changing the questionnaire logic and answer categories}

By applying gatekeeper logic, we eliminate the opportunity for athletes to report 'full participation without health problems' in question 1 and then (inconsistently) report the existence of a health problem in the subsequent questions. The revised questionnaire is, therefore, likely to reduce the number of health problems identified.

To estimate the extent to which this occurs, we calculated the number of health problems identified when gatekeeper logic was and was not applied, using a convenience sample of 13888 OSTRC-H responses from elite Norwegian athletes. As shown in table 4 , approximately $13 \%$ of the total number of cases were missed when gatekeeper logic was used. However, the missing cases were almost all of minor severity, given that $98.5 \%$ of substantial problems were still captured using gatekeeper logic.

\section{Additional questions}

The OSTRC questionnaire is a tool to capture and monitor health problems longitudinally in athletic populations. Following the four key questions, additional questions must be used to classify health problems and provide additional information to researchers or clinicians. We did not attempt to make recommendations on these follow-up questions, for a number of reasons. First, the questions used to classify health problems (eg, injured body part, injury type) should follow consensus-based recommendations. ${ }^{7}$ Second, the level of detail that is necessary and/or feasible to collect will vary between clinical and research settings. For example:

- Collecting free-text (qualitative) information about a health problem, or knowing exactly who is aware of it, may be unnecessary in research studies where investigators do not need to provide clinical responses based on athlete responses. However, this information can be valuable when clinicians use the questionnaire in a practical athlete-monitoring context.

- For some research questions, such as those investigating relationships between load and injury or those related to subsequent injuries, knowing the exact date of injury or symptom onset may be extremely important. 
- It may be relevant to record whether the athlete has not been able to participate due to other reasons than health problems, such as holidays, work or school commitments.

- Users cannot calculate the exact number of time-loss days from the four key questions alone. A follow-up question is needed to collect this information accurately.

Users should, therefore, customise additional questions of the OSTRC questionnaires to suit their research and/or clinical needs and should categorise data according to international classification standards, such as the IOC consensus statement on methods for recording and reporting epidemiological data on injury and illness in sports. ${ }^{7}$ Conversely, we recommend that users retain the exact wording and scoring of the four key questions to facilitate data interpretation, comparison and pooling.

\section{DISCUSSION}

In addition to reviewing the content and structure of the OSTRC questionnaires, the expert group discussed a range of general issues related to the questionnaire use, outcome measures, analysis methods and the need for consistent scientific reporting. Our aim was not to reach consensus on all topics, but to highlight areas that users may want clarified, share lessons learnt and to identify areas needing further research.

\section{Are two questionnaires necessary?}

As the OSTRC-H is designed to record all types of health problems, it may appear to negate the need for the OSTRC-O, which is limited to recording overuse injuries in predefined anatomical areas. However, previous research has shown that questionnaires asking athletes about specific injury types capture a greater number of problems in that location than when generalised questions are used. ${ }^{8}$ Therefore, the OSTRC-O may be preferable in studies that focus on overuse injuries to one specific injury location, particularly when collecting data for risk factor studies and randomised controlled trials. ${ }^{9-11}$ In these cases, competing risks should be considered in the statistical analyses to reduce the risk of bias. ${ }^{12}$

\section{Distribution frequency}

As the questionnaire refers to the previous 7 days, weekly distribution of the questionnaire is necessary to capture every health problem. However, for certain research questions, it may be acceptable to distribute the questionnaire less frequently, such as every second week ${ }^{9} 1013$ or every month. ${ }^{14}$ If investigators or clinicians choose this approach, some short-duration cases may not be recorded, but outcome measures will still be comparable to data collected weekly. ${ }^{15}$ We highlight, however, that to limit recall bias, ${ }^{15}$ even if the questionnaire is distributed infrequently (eg, only once a month), the questions should still refer to 'the past 7 days'.

In elite sports, it is becoming increasingly common to collect training-related data from athletes every day. In this case, daily monitoring of health status using a modified version of the OSTRC-H is also feasible. This may be clinically valuable if athletes have sufficiently intensive medical coverage and may allow for greater accuracy in the collected data. However, data collected daily may not be directly comparable to those collected weekly. This approach will also increase the demand on the athletes, and response rates and accuracy may decline over time.

\section{Outcome measures and analytical approaches}

Traditional epidemiological constructs, such as injury and illness incidence, prevalence and severity can be obtained using the
OSTRC questionnaires with some basic additional questions on exposure and time loss. In addition, because the OSTRC questionnaires provide details on the consequences and symptoms of health problems, a range of other outcome measures can also be presented. The severity score and substantial health problems are two novel outcomes proposed with the original OSTRC questionnaires. These measures may be valuable for clinical use; however, as they are yet to be fully validated, researchers must consider their limitations.

The severity score falls between 0 (full participation without health problem) and 100 (no participation at all) and is calculated based on the athlete's responses on the four OSTRC questions. This score allows the visualisation of changes in the consequences of health problems over time for an athlete, which can be valuable for coaches, athletes and clinicians. However, using the severity score appropriately in analyses can be problematic. Although the severity score has previously been analysed as a continuous outcome variable, we emphasise that it does not satisfy the basic requirements of a continuous measure (eg, equal interval between possible scores). In effect, therefore, the severity score represents an ordinal-scale variable with 25 possible outcomes, not 100 . Recent publications highlight the analytical benefits of representing various 'states' of an athlete's health on an ordinal scale. ${ }^{16}$ Unfortunately, for this approach to be feasible with small samples (as is normally the case in sports medicine research), the number of potential states needs to be far fewer than 25 to reduce the risk of sparse data bias. ${ }^{17}$ Using the time-varying nature of the OSTRC questionnaires outcomes in advanced multistate models is an area requiring further development and validation.

Of particular note is the longitudinal nature of the data registered with the OSTRC questionnaires. Longitudinal approaches provide opportunities to explore the risks and prognoses of health problems over time and account for correlated data. Such analyses are of special interest for elite athletic populations that, in general, are smaller in number but tend to experience multiple events (injuries and/or illnesses). When considering longitudinal analytical methods, users should be aware of the inherent challenges of these methods, including (i) missing data, (ii) timevarying exposures, outcomes, confounders, effect-measure modifiers and mediators, (iii) recurrent/subsequent events and (iv) competing risks. ${ }^{1618} 19$

\section{Factors that may promote athletes to adopt the OSTRC questionnaires}

As shown in our citation analysis, multiple authors have employed the OSTRC questionnaires since the first publication in 2013. ${ }^{1}$ This suggests that the questionnaires represent versatile and functional tools for both research and clinical use. In many cases, studies have reported high response rates from athletes. However, the questionnaires have not always been successfully adopted by athletes. ${ }^{2021}$ In our experience and based on recent research, ${ }^{21}$ factors that are crucial to obtaining high response rates in the clinical setting include providing feedback to respondents, timely follow-up by clinicians when a health problem is reported and personal interaction with athletes to motivate them. Similar factors influence the implementation of sports injury surveillance practices outside clinical settings. ${ }^{22}$

We recognised that to encourage adoption of the questionnaire, its name should be relevant and recognisable to respondents. Although researchers should report the official questionnaire titles and abbreviations (OSTRC-O2 and OSTRC$\mathrm{H} 2$ ) in scientific communications, it is not necessary to use the 


\section{What is already known on this topic}

- The Oslo Sports Trauma Research Center questionnaires were developed to address challenges in recording the full extent of sports-related health problems using standard sports injury data collection methods.

- The questionnaires have been widely adopted in sports injury research and in clinical health monitoring programmes by a range of elite sports organisations.

- Due to this successful uptake, a range of questionnairerelated issues requiring clarification or modification have been identified.

\section{What this study adds}

- This manuscript proposes specific changes to the wording, structure and logic of the Oslo Sports Trauma Research Center (OSTRC) questionnaires and discusses the likely impact of the proposed changes on collected data.

- Updated versions of the questionnaires (OSTRC-02 and OSTRC-H2) are provided.

- A range of general issues related to questionnaire use, outcome measures, analysis methods and implementation success are discussed, alongside areas needing further research.

official titles when delivering the questionnaire to respondents. Instead, we encourage users to rename the questionnaires to suit their purpose and context (eg, The Norwegian Olympic Team Health Report).

Importantly, this paper presents updates to the OSTRC questionnaires, so they provide greater clarity and consistency in the questions themselves. We also hope to ensure easier navigation using the gatekeeper logic. We believe that these changes will provide a better experience for the respondents and, in turn, maximise their adherence.

\section{Subjectivity and context specificity}

Completion of the OSTRC questionnaires requires a high level of subjectivity. Questions ask for the athletes' perceptions of the consequences that a health problem has on their participation and performance, as well as their perceived symptoms. These perceptions are dependent on contextual factors such as athlete experience, level of sports, type of sport and time of season. ${ }^{2324}$ This means that data collected from different cohorts of athletes will not necessarily be comparable. We encourage further research to explore the psychometric properties of the OSTRC questionnaires across different contexts and populations.

When the questionnaires are applied to contexts beyond adult sports (eg, among children, athletes with disability, performing artists and in occupational settings), the wording should be adjusted. We recommend an expert-based approach to ensure sound principles behind such adjustments, as we employed in this update. Any future adaptations of the questionnaire should undergo psychometric testing and validation.

\section{CONCLUSION}

Users from a range of sports research and clinical environments have gained experience using the OSTRC questionnaires and have identified areas in which they could be improved. In this paper, to provide greater clarity and consistency of questioning, we present changes to the wording, structure and logic of the original OSTRC questionnaires. We believe that these changes will improve athletes' experience when completing the questionnaires and also improve the quality of collected data.

\section{Author affiliations}

${ }^{1}$ Department of Sports Medicine, Norwegian School of Sport Sciences, Oslo Sports Trauma Research Center, Oslo, Norway

${ }^{2}$ LASEM Research Centre, La Trobe University, Bundoora, Victoria, Australia

${ }^{3}$ Athlete Availability Program, Australian Institute of Sport, Canberra, Australian Capital Territory, Australia

${ }^{4}$ Exercise Medicine Research Institute, School of Medical and Health Sciences, Edith Cowan University, Perth, Western Australia, Australia

${ }^{5}$ Family Practice \& Kinesiology, The University of British Columbia, Vancouver, British Columbia, Canada

${ }^{6}$ Southern California University of Health Sciences, Whittier, California, USA

${ }^{7}$ University of Western States, Portland, Oregon, USA

${ }^{8}$ School of Sport and Health Sciences, Cardiff Metropolitan University, Cardiff, UK

${ }^{9}$ Institute of Sports Science and Clinical Biomechanics, University of Southern

Denmark, Odense, Syddanmark, Denmark

${ }^{10}$ Sports Medicine Division, United States Olympic Committee, Colorado Springs, Colorado, USA

${ }^{11}$ Department of Public Health, Aarhus University, Aarhus, Denmark

${ }^{12}$ Sport Injury Prevention Research Centre (SIPRC), Faculty of Kinesiology, University

of Calgary, Calgary, Alberta, Canada

${ }^{13}$ Tampere Research Center of Sport Medicine, UKK Instituutti, Tampere, Finland

${ }^{14}$ Sports, Exercise, Medicine and Lifestyle Research Institute (SEMLI), University of Pretoria, Pretoria, South Africa

${ }^{15}$ Medical and Scientific Department, International Olympic Committee, Lausanne, Switzerland

${ }^{16}$ Department of Public and Occupational Health, Amsterdam University Medical Centres, Amsterdam, Noord-Holland, The Netherlands

Twitter Benjamin Clarsen @benclarsen, Stig Haugsboe Andersson @ stighandersson, Sean lain Docking @SIDocking, Michael Drew @_mickdrew, Caroline F Finch@CarolineFinch, Lauren Victoria Fortington @LFortington, Joar Harøy @JHaroey, Isabel S Moore @IzzyMoorePhD, Merete Møller @ Merete_Moller, Dustin Nabhan @nabhansportsmed, Rasmus Oestergaard Nielsen @RUNSAFE_Rasmus, Torbjørn Soligard @TSoligard and Evert Verhagen @ Evertverhagen

Acknowledgements The Oslo Sports Trauma Research Center has been established at the Norwegian School of Sport Sciences through generous grants from the Royal Norwegian Ministry of Culture, the South-Eastern Norway Regional Health Authority, the International Olympic Committee, the Norwegian Olympic Committee \& Confederation of Sport and Norsk Tipping AS. CFF, LVF and MD are members of the Australian Centre for Research into Injury in Sport and its Prevention (ACRISP) at Edith Cowan University. ACRISP is one of the International Research Centres for the Prevention of Injury and Protection of Athlete Health supported by the International Olympic Committee.

Contributors All authors were involved in the questionnaire review process, provided editorial input to the text and approved the final manuscript. BC and EV drafted the manuscript and performed the analyses. SHA performed the citation search.

Funding The authors have not declared a specific grant for this research from any funding agency in the public, commercial or not-for-profit sectors.

Competing interests None declared.

Patient consent for publication Not required.

Provenance and peer review Not commissioned; externally peer reviewed.

Data availability statement Data are available upon request.

\section{ORCID iDs}

Benjamin Clarsen http://orcid.org/0000-0003-3713-8938 Sean lain Docking http://orcid.org/0000-0001-7051-7548

Caroline F Finch http://orcid.org/0000-0003-1711-1930

Lauren Victoria Fortington http://orcid.org/0000-0003-2760-9249

Joar Harøy http://orcid.org/0000-0002-0475-637X

Karim M Khan http://orcid.org/0000-0002-9976-0258

Isabel S Moore http://orcid.org/0000-0002-4746-3390

Dustin Nabhan http://orcid.org/0000-0002-1244-515X

Rasmus Oestergaard Nielsen http://orcid.org/0000-0001-5757-1806

Kati Pasanen http://orcid.org/0000-0002-0427-2877

Torbjørn Soligard http://orcid.org/0000-0001-8863-4574

Evert Verhagen http://orcid.org/0000-0001-9227-8234 


\section{REFERENCES}

1 Clarsen B, Myklebust G, Bahr R. Development and validation of a new method for the registration of overuse injuries in sports injury epidemiology: the Oslo sports trauma research centre (OSTRC) overuse injury questionnaire. Br J Sports Med 2013:47:495-502.

2 Fuller CW, Ekstrand J, Junge A, et al. Consensus statement on injury definitions and data collection procedures in studies of football (soccer) injuries. Br J Sports Med 2006;40:193-201.

3 Bahr R. No injuries, but plenty of pain? on the methodology for recording overuse symptoms in sports. Br J Sports Med 2009;43:966-72.

4 Hammond LE, Lilley JM, Pope GD, et al. The impact of playing in matches while injured on injury surveillance findings in professional football. Scand J Med Sci Sports 2014;24:e195-200.

5 Clarsen B, Rønsen O, Myklebust G, et al. The Oslo sports trauma research center questionnaire on health problems: a new approach to prospective monitoring of illness and injury in elite athletes. Br J Sports Med 2014;48:754-60.

6 Jorgensen JE, Rathleff CR, Rathleff MS, et al. Danish translation and validation of the Oslo sports trauma research centre questionnaires on overuse injuries and health problems. Scand J Med Sci Sports 2016;26:1391-7.

7 Bahr R, Clarsen B, Derman W. International Olympic Committee consensus statement: methods for recording and reporting of epidemiological data on injury and illness in sport including STROBE extension for sport Injury and illness surveillance (STROBESIIS)). Br J Sports Med 2020;54:372-89.

8 Clarsen B, Krosshaug T, Bahr R. Overuse injuries in professional road cyclists. Am J Sports Med 2010;38:2494-501.

9 Andersson SH, Bahr R, Clarsen B, et al. Risk factors for overuse shoulder injuries in a mixed-sex cohort of 329 elite handball players: previous findings could not be confirmed. Br J Sports Med 2018;52:1191-8.

10 Andersson SH, Bahr R, Clarsen B, et al. Preventing overuse shoulder injuries among throwing athletes: a cluster-randomised controlled trial in 660 elite handball players. Br J Sports Med 2017;51:1073-80.
11 Harøy J, Clarsen B, Thorborg K, et al. Groin problems in male soccer players are more common than previously reported. Am J Sports Med 2017;45:1304-8.

12 Andersen PK, Geskus RB, de Witte T, et al. Competing risks in epidemiology: possibilities and pitfalls. Int J Epidemiol 2012:41:861-70.

13 Clarsen B, Bahr R, Andersson SH, et al. Reduced glenohumeral rotation, external rotation weakness and scapular dyskinesis are risk factors for shoulder injuries among elite male handball players: a prospective cohort study. Br J Sports Med 2014;48:1327-33.

14 Pluim BM, Clarsen B, Verhagen E. Injury rates in recreational tennis players do not differ between different playing surfaces. Br J Sports Med 2018;52:611-5.

15 Valuri G, Stevenson M, Finch C, et al. The validity of a four week self-recall of sports injuries. Inj Prev 2005;11:135-7.

16 Nielsen RO, Bertelsen ML, Ramskov D, et al. Time-to-event analysis for sports injury research Part 2: time-varying outcomes. Br J Sports Med 2019;53:70-8.

17 Greenland S, Mansournia MA, Altman DG. Sparse data bias: a problem hiding in plain sight. BMJ 2016;352:i1981.

18 Nielsen RO, Bertelsen ML, Ramskov D, et al. Time-to-event analysis for sports injury research Part 1: time-varying exposures. Br J Sports Med 2019;53:61-8.

19 Ullah S, Gabbett TJ, Finch CF. Statistical modelling for recurrent events: an application to sports injuries. Br J Sports Med 2014;48:1287-93.

20 Bromley S, Drew M, Talpey S, et al. Collecting health and exposure data in Australian Olympic combat sports: feasibility study utilizing an electronic system. JMIR Hum Factors 2018;5:e27.

21 Barboza SD, Bolling CS, Nauta J, et al. Acceptability and perceptions of end-users towards an online sports-health surveillance system. BMJ Open Sport Exerc Med 2017:3:e000275.

22 Ekegren CL, Donaldson A, Gabbe BJ, et al. Implementing injury surveillance systems alongside injury prevention programs: evaluation of an online surveillance system in a community setting. Inj Epidemiol 2014;1:19.

23 Bolling C, Delfino Barboza S, van Mechelen W, et al. How elite athletes, coaches, and physiotherapists perceive a sports injury. Trans/ Sports Med 2019;2:17-23.

24 Bolling C, van Mechelen W, Pasman HR, et al. Context matters: revisiting the first step of the 'sequence of prevention' of sports injuries. Sports Med 2018;48:2227-34. 